\title{
Hyperspectral Monitoring of Fructose Content in Marzipan
}

\author{
KATALIN BADAK-KERTI ${ }^{*}$, SZABINA NÉMETH, ANDREAS ZITEK², \\ FERENC FIRTHA ${ }^{3}$
}

\begin{abstract}
In our research marzipan samples of different sugar to almond paste ratios $(1: 1,2: 1,3: 1)$ were stored at $17^{\circ} \mathrm{C}$. Reducing sugar content was measured by analytical method, texture analysis was done by penetrometry, electric characteristics were measured by conductometry and hyperspectral images were taken 6-8 times during the 16 days of storage. For statistical analyses (discriminant analysis, principal component analysis) SPSS program was used.

According to our findings with the hyperspectral analysis technique, it is possible to identify how long the samples were stored (after production), and to which class (ratio of sugar to almond) the sample belonged. The main wavelengths which gave the best discrimination results among the days of storage were between 960 and $1100 \mathrm{~nm}$. The type of the marzipan was easy to distinguish with the hyperspectral data; the biggest differences were observed at 1200 and $1400 \mathrm{~nm}$, which are connected to the first overtone of $\mathrm{C}-\mathrm{H}$ bound, therefore correlate with the oil content. The spatial distribution of penetrometric, electric and spectral properties were also characteristic to fructose content.

The fructose content of marzipan is difficult to measure by usual optical ways (polarimetry, spectroscopy), but since fructose is hygroscopic, the spatial distribution of spectral properties can be characteristic.
\end{abstract}

Keywords: hyperspectral analysis, marzipan, fructose content

\section{Introduction}

Marzipan is a delicacy which is made primarily from almond and sugar. In some countries it can contain other nuts (e.g. apricot kernel) as well.

\footnotetext{
* Corresponding author. E-mail: badakne.dr.kerti.katalin@etk.szie.hu

1Szent István University, Dep. of Grain and Industrial Crop Technology, Villányi út 29-43, 1118 Budapest, Hungary

${ }^{2}$ Institute of Wood Technology and Renewable Materials, Department of Material Sciences and Process Engineering, University of Natural Resources and Life Sciences Vienna BOKU, Peter Jordan Str.82, A-1190 Vienna, Austria

${ }^{3}$ Szent István University, Dep. of Physics and Automation, Somlói út 16, 1118 Budapest, Hungary
} 
Blanched almonds are mixed with sugar and glucose or sugar and invert syrup mixtures in different sugar to almond ratios. For its significant taste bitter almonds are also used in the production (up to $1 \%$ of the total nut weight should be bitter). Based on the Codex Alimentarius Hungaricus the marzipan products are classified according to their sugar to almond ratios, where the sugar content (expressed in sucrose, on dry base) is 35, 50, 67 or $75 \mathrm{w} / \mathrm{w} \%$.

Although during the ageing of marzipan the total sugar content is not altering, the ratio of the different sugar components changes. Invertase enzyme transforms the non-reducing sucrose into glucose and fructose molecules. (Chandrasekaran, 2015)

Unlike saccharose (sucrose), fructose is highly hygroscopic; therefore the drying of the marzipan is reduced.

The reducing sugar content (fructose) is measured in general by titrimetric or iodometric analytical methods (e.g. the Schoorl Regenbogen method) (Mohos, 2010), which are time and solvent consuming, and require analytical skills.

Other techniques such as HPLC and ion chromatography are also used in direct sugar analysis. However, to use these methods the sample preparation is crucial, as most methods of sugar analysis require a clean aqueous extract to work on (Edwards, 2000), and in marzipan sugar is present in a matrix of lipid and protein.

Christensen et al. used in their study a set of 32 marzipan samples of nine different recipes to measure their composition with different types of NIR spectrometers. The marzipan samples were analyzed chemically and measured on six different spectrometer set-ups. Partial least squares regression (PLSR) method was used to make calibrations to moisture and sugar content. In their model the root mean square error of cross-validation (RMSECV) for the prediction of the moisture was found to be $0.36 \% \mathrm{w} / \mathrm{w}$ moisture (range 7-19\%). The sugar content was predicted with a RMSECV of $1.43 \% \mathrm{w} / \mathrm{w}$ (range $33-68 \%$ ). (Christensen et al., 2004)

The same set was used for the work of Westad et al. (2007), where relevant wavelengths data were searched for the best differentiation. Sugar and water constitute the two major chemical components in the marzipan data, and as expected the spectral pattern reveals a distinction between $\mathrm{O}-\mathrm{H}$ vibrations (sugar and water) and $\mathrm{C}-\mathrm{H}$ vibrations (sugar). For instance, the broad water bands in the NIR regions at 800-1100 nm (O-H stretch, 2. overtone) and around $1900 \mathrm{~nm}$ (O-H stretch/deformation combination) are well correlated to the $\mathrm{O}-\mathrm{H}$ deformation band in the $700-800 \mathrm{~cm}^{-1}$ 
(12 500-14 $286 \mathrm{~nm}$ ) region of the IR spectra. The $1200 \mathrm{~nm}$ NIR region (C-H stretch, 2. overtone), on the other hand, show significant correlations to IR bands around for instance $820 \mathrm{~cm}^{-1}$ (C-H deformation, $12200 \mathrm{~nm}$ ) and 1030 $\mathrm{cm}^{-1}(\mathrm{C}=\mathrm{O}$ stretch, $9700 \mathrm{~nm})$. Last, the NIR bands around 1400-1500 $\mathrm{nm}$ are related to both $\mathrm{O}-\mathrm{H}$ stretch vibrations and $\mathrm{C}-\mathrm{H}$ combinations. (Westad, Afseth and Bro, 2007)

Hyperspectral imaging has been also used on different food products; for example to measure the moisture content of carrots (Firtha, 2008 and 2007), ham (Talens et al, 2003) and soy beans (Huang et al, 2013). The same technique was used on plants, too. (Jung, Kardeván and Tókei, 2006)

The aim of the present study was to evaluate the feasibility of hyperspectral imaging technique, conductometry and penetrometry to follow the changes induced by altering fructose content of marzipan during storage.

\section{Materials and Methods}

Marzipan samples of 3 different sugar to almond ratios (1:1, 2:1, 3:1) were produced under the same manufacturing parameters. Prismatic samples (rods) of the same geometry were cut out of the marzipan blocks. Samples were stored at constant temperature of $17^{\circ} \mathrm{C}$ in a closed cabinet. Storage lasted 16 days after production, as this time is enough to finish the sucrose inversion.

Measurements were taken on day 1, 3, 7, 10, 14, 16 after production.

Reducing sugar content was measured by the Schrool Regenboogen method according to the Hungarian standard MSZ 20900/5-1989.

Hyperspectral imaging were done using HeadWall push broom system (900-1700 nm range, 256*320 InGaAs sensor matrix, 14 bit A/D, $800 \mathrm{~nm} / 155$ band $=5.16 \mathrm{~nm} /$ px spectral resolution, $214^{*} 100 \mathrm{~mm}$ : $686^{*} 320$ px image size, $100 \mathrm{~mm} / 320 \mathrm{px}=312 \propto \mathrm{m} / \mathrm{px}$ spatial resolution, $45^{\circ} / 0^{\circ}$ illumination geometry, HeadWall Photonics, Inc, Fitchburg, MA, USA) and Argus calibration and controlling software (Firtha, 2010). The hyperspectral method measures the reflectance (reflection ratio).

Texture analyser Ta-Xt2 was used for penetrometric texture analyses. The maximal force (rupture point) was measured along drying rod at 7 points during 4 weeks of storing.

Conductivity of the samples was measured with HP4284A Precision LCR Meter that acquires data in $20 \mathrm{~Hz}$ to $1 \mathrm{MHz}$ test frequency range. Tests 
were made on 48 selected frequencies at 7 points of drying rod during storage time.

For statistical analyses discriminant analysis and principal component analyses were used in SPSS 14.0 program.

\section{Results and Discussion}

During the storage of the marzipan samples as expected sucrose inverted into glucose and fructose, as it can be followed in Fig. 1. The speed of conversion was the highest in the first 3 days of storage. In case of a 2 sugar to 1 almond sample (67\% of sugar in the recipe) it seems, that the conversion did not finish after 16 days (as it seem that the reducing sugar content is still increasing), which is not in harmony with the literature data. At the end of the storage, the reducing sugar content has reached a maximum of $14.3 \%$ in case of 1 sugar to 1 almond marzipan and a maximum of $9.5 \%$ in 3 sugar to 1 almond marzipan.

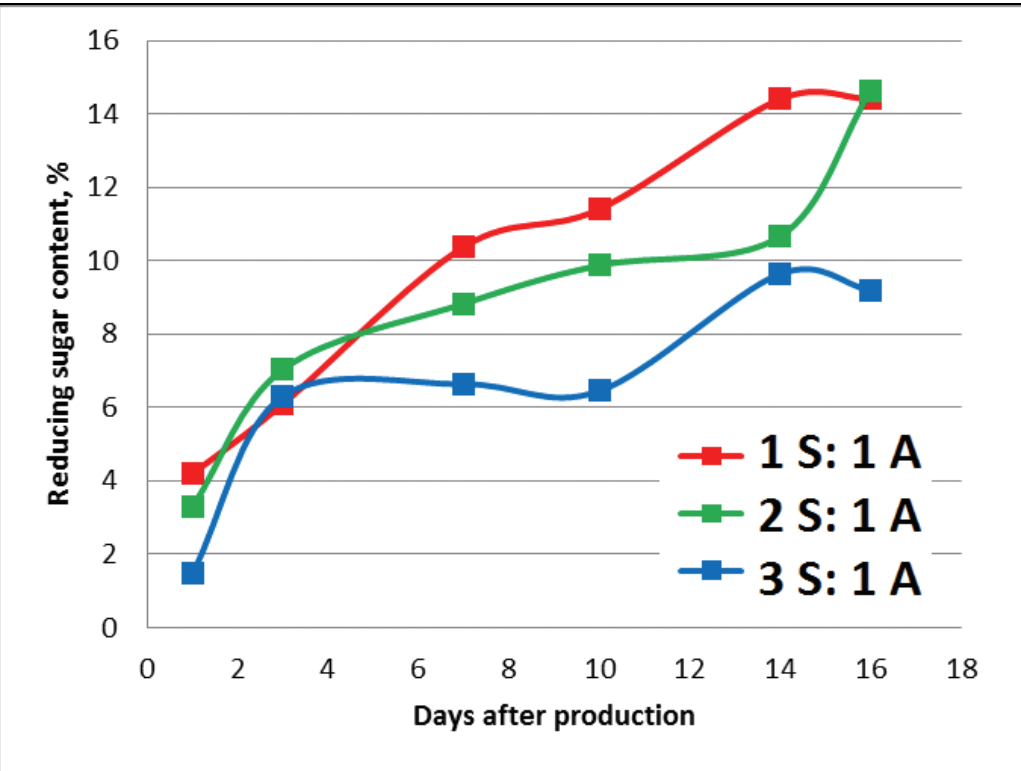

Fig. 1. Changing of the reducing sugar content during storage of different marzipan samples (ratios of $\mathrm{S}$ - sugar, A - almond) 


\subsection{Relationship between storage time and hyperspectral data}

The images of the marzipan prisms were divided into 7 regions. The spectra of pixels were averaged and saved for each area. This way the spatial distribution of reflectance spectra were measured. The spectra were normalized, but finally the first derivatives were optimal for building chemometric modell. The derivatives were calculated by Savitzky-Golay method (order $=3$, length $=9$ ) as described earlier by Firtha (2010). These data were then used for further analysis to define correlations between storage time and hyperspectral data and type of marzipan and spectral data.

In the first analysis the correlation was studied between the day of storage and the spectral data, not taking into account which sugar to almond ratio the marzipan sample had. During the storage of marzipan the shape of the spectra have not changed significantly, independently from the type of marzipan. The measured values on day 3 were outliers, most probably due to a measurement failure, and therefore those values were removed from further analyses.

Using the discriminant functions a classification was done, where $88.6 \%$ of the original grouped cases were correctly classified, and using randomly selected data a cross validation also resulted in a good classification ( $83.8 \%$ of sample groups were correctly classified).

The discriminant function coefficients show that the lower wavelenghts (960-1100 nm) are the most useful to define how many days have passed after production.

\subsection{Relationship between sugar to almond ratios and hyperspectral data}

Figure 2 shows the spectral data of the different marzipan samples after the first and sixteenth day of storage. The biggest differences can be seen around $1200 \mathrm{~nm}$, which is the wavelength where oils are absorbing the most light according to the literature data. In the marzipan which had the same amount of sugar and almond, the oil content was the highest among the samples, so its reflectance was the biggest on this wavelength. Above 1400 $\mathrm{nm}$ the spectra of the 1 sugar to 1 almond sample is smooth, where the other samples show peaks. According to literature data in this region sugars and water significantly form the reflectance values. (Burns and Ciurczak, 2007) 

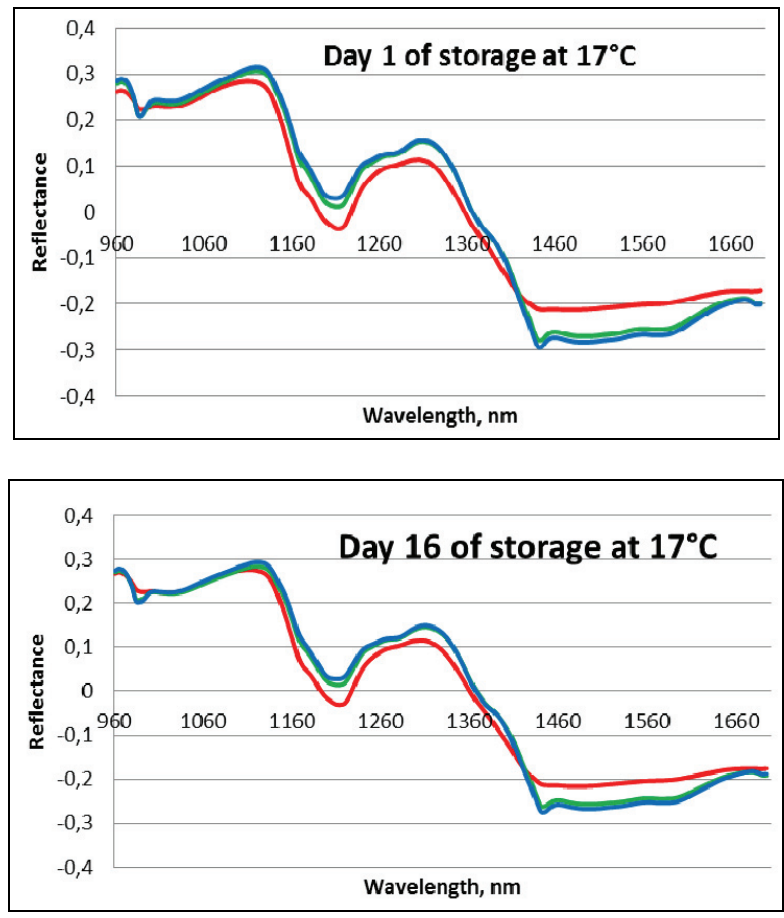

Fig. 2. Reflectance spectra of marzipan samples on day 1 and day 16 after production, red line 1S:1A, green line $2 \mathrm{~S}: 1 \mathrm{~A}$, blue line $3 \mathrm{~S}: 1 \mathrm{~A}$, where $\mathrm{S}$ is sugar, $\mathrm{A}$ is almond, ratios on dry matter base

With discriminant analysis a clear classification could be done among sample groups according to their sugar content. In the case of marzipan samples 2 and 3 (2 sugar to 1 almond and 3 sugar to 1 almond ratios) the group centroids are closer to each other than in the case of sample 1 (1 sugar to 1 almond). Along canonical function 1 better discrimination has been reached than along function 2 . There was a $100 \%$ success in the classification of the groups, also in cross-validation.

In this analysis not only the earlier mentioned $1200 \mathrm{~nm}$ (oil content related region) can be found as a significant wavelength for the discrimination, but also the lower regions (960-1000 nm) are important factors.

Using discriminant analysis we could distinguish among the marzipan samples of different sugar contents. With principal component analysis (PCA) we identified the wavelengths which gave the biggest significant 
differences between the studied samples (Fig. 3). Wavelengths of whose weight is above 0.25 have a significant effect in the components of the discrimination in PCA. These wavelength ranges are 960-1160 nm and $1430-1610 \mathrm{~nm}$. It is interesting to see that the signs of each component are changing at the wavelengths which were mentioned earlier in the discriminant analysis.

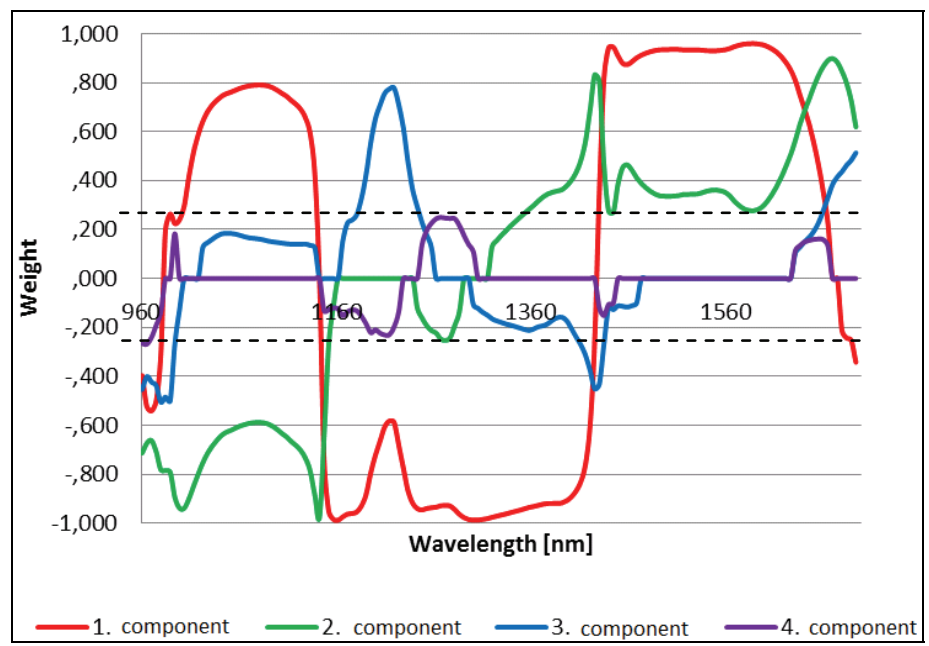

Fig. 3. Weights of the wavelengths as independent variables in PCA

\subsection{Discussion of the penetrometric, electric and hyperspectral data}

Hardness of marzipan can be described as the maximum force needed to rupture the surface of the sample. Conductivity of marzipan is directly connected to the bound state of water. In Fig. 4 three graphs can be seen representing the hardness, the impedance and the reflectance values of the marzipan rods, measured 2 times a week during a 4-week storage period. On the $\mathrm{x}$ axis the dimension of the marzipan rod is described. 0 in this case means the middle point of the rod. Although the force/impedance/spectral values do not give an exact representation of the fructose content, the spatial distribution of the data shows how the sides are drying out more than the inner parts of the rods. In the middle of the rods, where the fructose could withhold the drying out of marzipan, hardness remained 
lower, impedance of the samples were smaller and the spectral data showed also lower values at $1450 \mathrm{~nm}$, which is the region connected to water.

Maximal force (rupture point) along drying rod

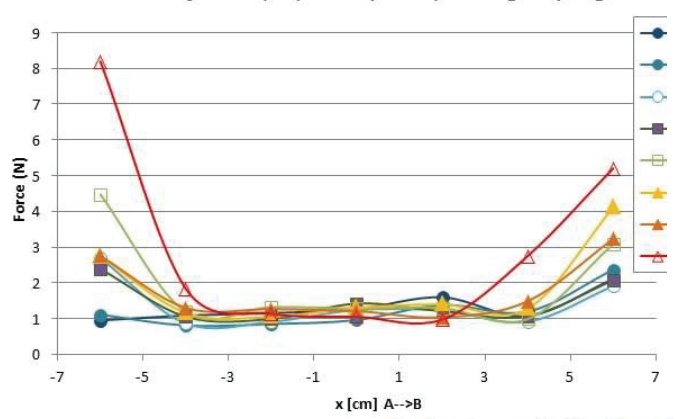

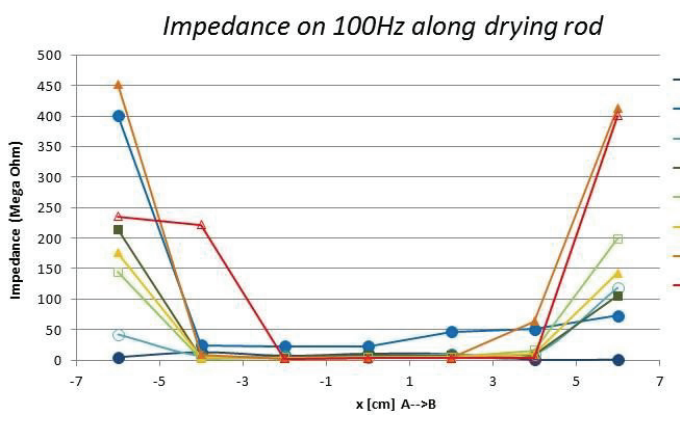

Reflectance (reflection factor) at $1450 \mathrm{~nm}$ along drying rod [cm]

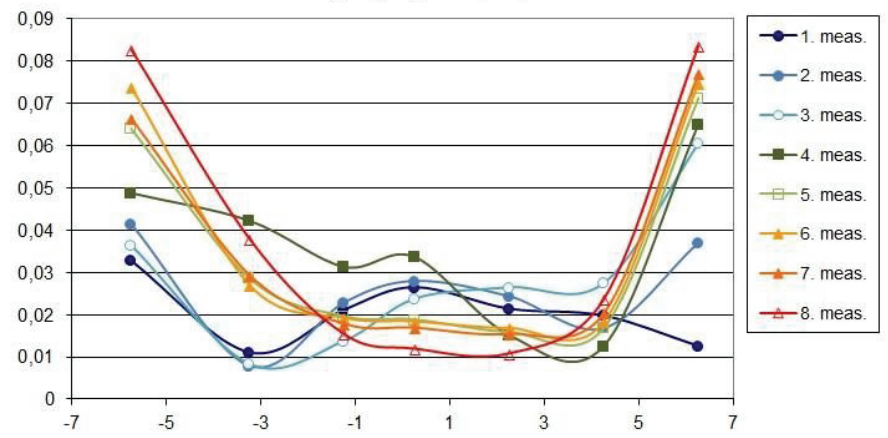

Fig. 4. Hardness (maximal force), impedance and reflectance at $1450 \mathrm{~nm}$ of marzipan rods during storage at $17^{\circ} \mathrm{C}$, represented as measured on the surface of a marzipan rod from side to side

Measuring fructose content of marzipan by optical methods faces many obstacles. Polarimetry is not of use, because there are more sugar components and therefore the diffuse reflection of the surface disperses the polar plane.

Spectroscopy is also not suitable, because one can differentiate only between monosaccharides and disaccharides, but not between sugar type as glucose and fructose (both are monosaccharides, with similar spectra).

However, as the fructose is hygroscopic, it attracts and binds water, the spatial distribution of properties, which are depending on the water content (like absorption at $1450 \mathrm{~nm}$ ), is characteristic. 
The spatial distribution of the moisture content of a drying wall from a normal material is cosine-like (see Fourier solution of unsteady heat transfer in a wall). In case of hygroscopic material, like fructose, the spatial distribution is constant in the middle and changes on the edges only. This can be followed by hyperspectral method, so it allows checking the fructose content of a marzipan sample.

\section{Conclusions}

Hyperspectral imaging has the advantage that samples can be measured without polluting them. Although this measurement cannot give exact information about the fructose content of marzipan, the spatial distribution of the electric, penetrometric and hyperspectral data were similar.

With discriminant analyses we were able to make classifications according to the sugar content of the marzipan and it was possible to distinguish among the days of storage as well. The fructose content of marzipan cannot be measured by usual optical ways (polarimetry, spectroscopy), but since fructose is hygroscopic, the spatial distribution of spectral properties can be characteristic.

Further research is needed to check if hyperspectral technique can be used on packed samples, too.

\section{References}

Burns, D.A. and Ciurczak, E.W. (eds) (2007). Handbook of near-infrared analysis. CRC Press, Boca Raton, USA

Chandrasekaran, M. (2015). Enzymes in food and beverage processing. CRC Press, Boca Raton, USA

Christensen, J., Nørgaard, L., Heimdal, H., Pedersen, J.G. and Engelsen, S.B. (2004). Rapid spectroscopic analysis of marzipan - Comparative instrumentation. Journal of Near Infrared Spectroscopy, 12: 63-75.

Edwards, W.P. (2000). The science of sugar confectionery. Royal Society of Chemistry, Cambridge, UK

Firtha, F. (2007). Development of data reduction function for hyperspectral imaging. Progress in Agricultural Engineering Sciences,3(1): 67-88.

Firtha, F (2010): Argus and Cubrowser algortithms. ftp:/ / fizika2.kee.hu/FFirtha/ ArgusCubrowser.pdf (Accessed 20 April 2015).

Firtha, F., Fekete, A., Kaszab, T., Gillay, B., Nogula-Nagy, M., Kovács, Z. and Kántor, D. (2008) .Methods for improving image quality and reducing data load of NIR hyperspectral images. Sensors-Basel, 8:, 3287-3298. 
Huang, M., Wang, Q., Zhang, M. and Zhu, Q. (2013). Prediction of color and moisture content for vegetable soybean during drying using hyperspectral imaging technology. Journal of Food Engineering 128: 24-30.

Jung, A., Kardeván, P. and Tókei, L (2006). Hyperspectral technology in vegetation analysis. Progress in Agricultural Engineering Sciences, 2(1): 95-117.

Mohos, F. (2010). Confectionery and chocolate engineering: Principles and applications. John Wiley \& Sons, Chichester, UK

Talens, P., Mora, L., Morsy, N. and Da-Wen Sun, L. (2013). Prediction of water and protein contents and quality classification of Spanish cooked ham using NIR hyperspectral imaging. Journal of Food Engineering, 117(3): 272-280.

Westad, F., Afseth, N.K. and Bro, R. (2007). Finding relevant spectral regions between spectroscopic techniques by use of cross model validation and partial least squares regression, Analytica Chimica Acta, 595: 323-327. 